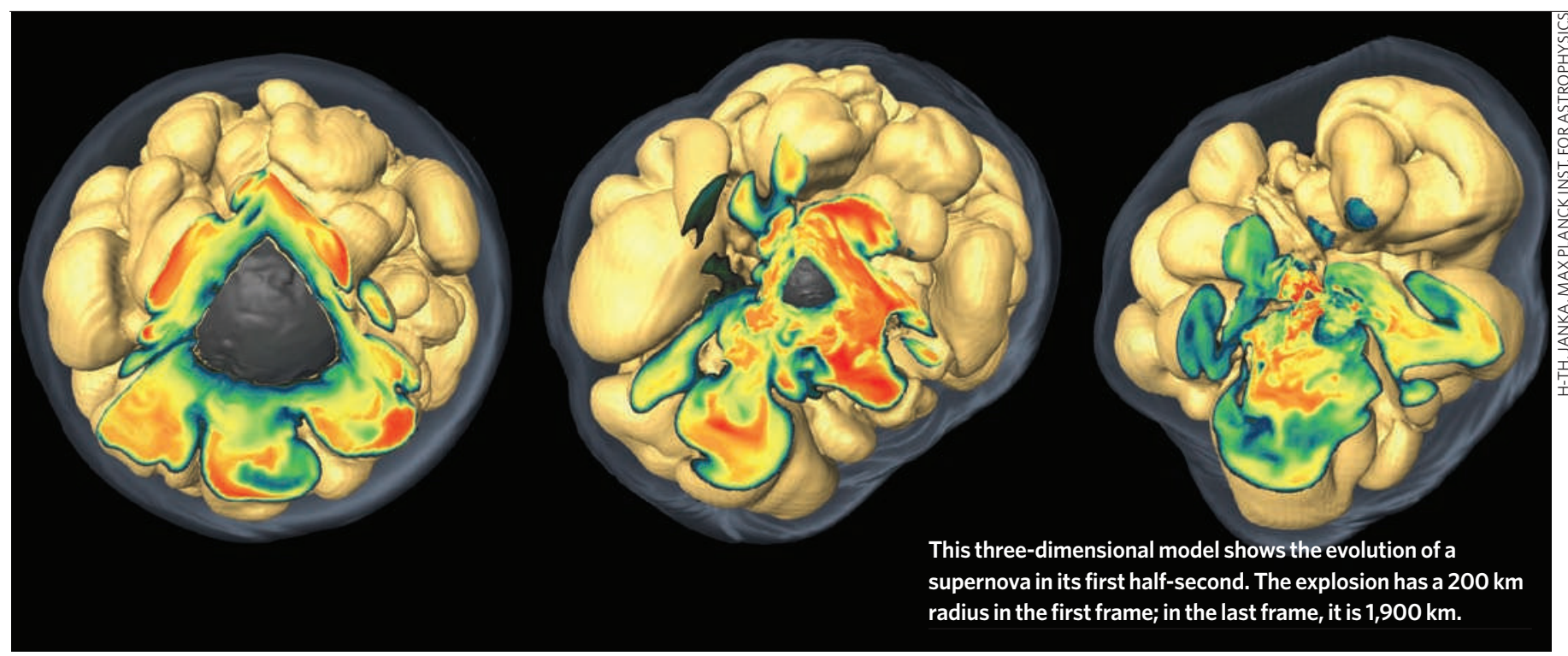

\title{
Model stars set to explode
}

\section{Realistic computational models of supernovae might soon solve a long-standing mystery.}

Giant, mushroom-shaped blobs of nickel speed out from the blistering core of the supernova at 4,000 kilometres per second, piercing the smooth, ballooning sphere of hydrogen. The effect looks something like a cosmic hernia, but astrophysicist Hans-Thomas Janka couldn't be more pleased with what his computer model is showing.

In a paper published in the 10 May issue of the Astrophysical Journal ${ }^{1}$, Janka and his colleagues from the Max Planck Institute for Astrophysics in Garching, Germany, used their model to address a long-standing puzzle: how do heavier elements, synthesized in the core of the massive, dying star, get out of the explosion before the lighter stuff that sits in the star's outer shells?

But the simulated explosion, with its colourful protrusions, is significant for another reason. It marks the first published, nearly complete, three-dimensional (3D) model of a supernova. Simpler one-dimensional (1D) and two-dimensional (2D) models, which assume that an explosion unfolds symmetrically, fail to get ticking star bombs to blow. So a handful of computational astrophysics groups, including Janka's, are moving up to the third dimension. The computational demands of tracking a complex and rapidly evolving explosion in which densities, temperatures and velocities are all at physical extremes are themselves astronomical. And the researchers have yet to tackle the toughest challenge: modelling the first milliseconds of the explosion in the innermost core of a supernova.

But when they do, astrophysicists hope their models will finally reveal the root mechanism that triggers supernovae, a problem that has eluded their profession since modelling began 40 years ago. "I think there's overwhelming evidence that going to $3 \mathrm{D}$ calculations makes the explosions easier," says Eliot Quataert, who has been following progress in the field as director of the Theoretical Astrophysics Center at the University of California, Berkeley. "That's what everyone is rushing after now."

Supernovae are among the most energetic events in the Universe, briefly outshining entire galaxies as they synthe-

size and spread the heavy elements that are essential to biological life. One type of supernova detonates when a dense white-dwarf star sucks in enough additional mass for a fusion explosion. The second, and more mysterious, type occurs when a star a few times more massive than the Sun runs out of nuclear fuel. As the star's internal pressure plummets, its massive core collapses into a neutron star just a few tens of kilometres across. At this point the neutrons can be compressed no further, and the core rebounds, sending a shock wave out against the crushing weight of the star's outer layers. But the shock wave by itself isn't enough to blow the star apart, and for a fraction of a second it stalls. The question is what 'revives' the shock, causing the explosion to proceed.
Some have suggested that energy locked up in the tightly coiled magnetic fields of a spinning core might provide the extra nudge. But many theorists favour a trigger from neutrinos, which are produced in unimaginably large numbers when the neutron star is born. Because of their weak coupling with other forms of matter, the vast majority of the neutrinos simply fly straight through the dying star. But if enough impart their energy to the infalling matter, they could provide the extra kick needed to restart the shock wave and blow up the outer shells of matter. "We're at the point now where we believe the final threshold is being crossed for the neutrino model," says astrophysicist Stan Woosley at the University of California, Santa Cruz, who is leading a 3D modelling effort. "A lot of us think it's going to work when we take this next step."

\section{Multi-dimensional models}

But modelling the interactions of the neutrinos with the infalling matter is the most computationally difficult part of the problem; until now, modellers have had to use lower-dimensional short cuts. Not only have these models generally failed to ignite the supernova, but they also preserve unrealistic symmetries, with the progenitor star maintaining its onion layering as it blows up: hydrogen and helium first, followed by heavier elements. The spectacular 1987 supernova in the Large Magellanic Cloud 
was heralded by a burst of X-rays and $\gamma$-rays coming from radioactive heavy elements such as nickel - meaning that the heavy material had somehow punched through the lighter layers. "That was the point when theorists realized that $1 \mathrm{D}$ models cannot explain the physics that was observed in the explosion," says Janka.

The rapid increase in available computer power now allows the modellers to run more-ambitious and realistic simulations in a manageable time - currently, Janka needs two to three months to run one supernova in 3D. At some point, researchers say, a digital cataclysm will attain a level of detail adequate to reproduce what occurs in the real Universe. "That is what the big competition is about," says Janka.

\section{Optimistic outlook}

Adam Burrows, an astrophysicist at Princeton University in New Jersey, who is working with Woosley, has already analysed the benefits of 3D models. In 2008, he showed that going to 2D made a simulated supernova of a given luminosity blow up 1.4 times as often as the $1 \mathrm{D}$ version ${ }^{2}$. Repeating the analysis, he found that $3 \mathrm{D}$ models should make supernovae go off twice as easily as in 1D models. The reason, he says, is that $3 \mathrm{D}$ allows the infalling matter to take random walks in all directions - which means that it spends slightly more time interacting with neutrinos and absorbing their energy. Ultimately, the aim is to construct a 3D explosion that is faithful enough to generate a supernova without fail. In the process, the physical forces that govern it should become apparent.

That's why Janka is rushing to fill out the rest of his 3D model to include the neutrino mechanics in the first second of the explosion. He also has to find a computer that can handle the job. He estimates that he needs a computer to perform about $10^{21}$ floating point operations, or flops - roughly 50,000 times the computing power behind his latest simulations. This would mean several months of devoted time from a major supercomputer that can perform a petaflop, or $10^{15}$ operations, per second. With the growth in worldwide computing infrastructure, Quataert says, that is now not a completely outrageous request. "That's part of the reason for optimism that there will be a breakthrough in this problem," he says. Eric Hand

1. Hammer, N. J., Janka, H.-Th. \& Müller, E. Astrophys. J. 714, 1371 (2010).

2. Murphy, J. W. \& Burrows, A. Astrophys. J. 688, 1159 (2008)

\section{US prepares for climate burden}

It hasn't always been easy to get the White House to lead on climate change, so for years the question of how to incorporate global warming into long-range planning and public infrastructure in the United States has fallen to cities, states and individual federal agencies. Now, the Obama administration is looking to fold these independent efforts into a comprehensive adaptation strategy for the entire country.

Last week, about 150 experts gathered in Washington DC to swap ideas and information about exisiting adpatation plans across the country and to consider how the federal government should coordinate and encourage further steps. Ordered last year by John Holdren, Obama's chief science adviser, the three-day National Climate Adaptation Summit served as a brainstorming session where users and providers of 'climate services' could talk about their needs and capabilities. The gathering took place just a week after the National Research Council called on the government to develop a national strategy for dealing with the impacts of a changing climate.

"There's a sense that this is a moment to put everything together and figure out what we are going to do to prepare," says Richard Moss, a senior scientist at the Joint Global Change Research Institute in College Park, Maryland, and former director of the US Global Change Research Program. "And I give the administration credit for holding this meeting and asking for ideas, rather than just rolling out an answer."

Increased drought, heatwaves, forest fires, severe storms and rising sea levels in coastal cities are among the challenges that climate change could pose for the United States. A National Climate Service, modelled on the National Weather Service at the National Oceanic and Atmospheric Administration (NOAA) in Silver Spring, Maryland, could help local authorities to anticipate such stresses and provide a onestop clearinghouse for information designed to reduce susceptibility to climate-related disasters. While seeking congressional approval for the service, NOAA is pushing forwards with a reorganization that would essentially make the approach a reality. The plan includes a series of regional climate centres to help provide guidance for local governments and businesses.

The goal, says Thomas Karl, interim director of NOAA's Climatic Service, is to provide useful information about environmental impacts so that planners can assess water supplies, update flood-plain maps or decide how high to build levies. But the first step, he says, is to establish "good communication with those who will want to use the data”.

To be effective, the centres will need to be adept at translating hard data into metrics and standards to guide public and private infrastucture projects, says Anne Choate, vice-president of the business consultancy ICF International. "There's this gap between what the engineers say they need and what climate scientists can provide," she says.

Some scientists have already begun closing that gap. In 2008, a team led by Don Wuebbles, an atmospheric scientists at the University of Illinois at Urbana-Champaign, analysed global climate models to produce more detailed climate projections for the city of Chicago. The results suggest that the city could be significantly warmer in 2100 , with implications for everything from power generation to the kinds of tree that the city is planting today.

"We're going to look more like New Orleans than Chicago," says Joyce Coffee, who handles the city's adaptation planning. "We need to be planting species that will survive current cold snaps and thrive in a much warmer climate in the future."

Other federal initiatives are focusing on environmental planning at the regional level. For instance, the Interior Department is setting up 'Landscape Conservation Cooperatives' to bring federal agencies together with state and local governments to collaborate on planning for issues ranging from wildfires and water supplies to invasive species and energy development. "I think this is going to be the new model for environmental adaptation strategies," says Deputy Interior Secretary David Hayes.

An adaptation task force formed by the White House last year will incorporate the results of the summit into a white paper scheduled for release this autumn. Jeff Tollefson 